\title{
Brightfield Microscopy
}

National Cancer Institute

\section{Source}

National Cancer Institute. Brightfield Microscopy. NCI Thesaurus. Code C19827.

Brightfield microscopy is a type of light microscopy in which objects are seen against a bright background. 